\title{
The Efficacy of Treating Pulmonary Fibrosis and Pulmonary Function Injury in COVID-19 with the Fuzheng Huayu Tablets: study protocol for a multicenter randomized controlled trial
}

Fei Jing

Shuguang Hospital

Haina Fan

Shuguang Hospital

Zhimin Zhao

Shuguang Hospital

Feng Xing

Shuguang Hospital

Yingchun He

Shanghai University of Traditional Chinese Medicine

Chenghai Liu ( $\nabla$ chenghailiu@hotmail.com )

Shuguang Hospital

Study protocol

Keywords: Fuzheng Huayu Tablets, Pulmonary Fibrosis, Pulmonary Function Injury, COVID-19

Posted Date: July 2nd, 2020

DOI: https://doi.org/10.21203/rs.3.rs-37422/v1

License: (c) (i) This work is licensed under a Creative Commons Attribution 4.0 International License. Read Full License 


\section{Abstract}

Background: Some patients with COVID-19 have been found pulmonary dysfunction and/or fibrosis in the recovery period, especially severe cases, but there are no certain drugs or treatment to cope with this situation. Previous studies proved the efficacy of FZHY on lung fibrosis induced by Bleomycin in animals and improvement of pulmonary function in COPD patients. We design this trial to carry out the clinical study that the effects of FZHY Tablets on pulmonary fibrosis and/or pulmonary function injury in the recovery period of COVID-19 and expect to improve the prognosis.

Methods/design: This is a double-blind, placebo-controlled, randomized, multicenter clinical trial. It enrolls 160 patients who had been diagnosed with COVID-19, but currently they are negative for viral testing and have developed pulmonary fibrosis or pulmonary dysfunction. They are randomly divided equally into control group and experimental group. All patients are given basic treatment such as respiratory function rehabilitation training and vitamin $\mathrm{C}$. The control group is given placebo of FZHY, and the experimental group is given FZHY. Each patient will be observed for 24 weeks and followed up for 8 weeks. The primary outcome for the trial is a composite endpoint consisting of lung function and HRCT. Secondary outcomes include clinical symptoms, oxygen saturation and quality of life assessment.

Discussion: The trial is designed to test the hypothesis that treating pulmonary fibrosis or pulmonary dysfunction after SARS-CoV-2 infection with FZHY will improve the patient's lung function or the pathological manifestation of pulmonary fibrosis, and improve the quality of life.

Trial registration: Clinical Trials.gov, ID: NCT04279197. Registered on 12 April 2020.

\section{Background}

On February 11, 2020, World Health Organization (WHO) announced in Geneva that pneumonia infected with severe acute respiratory syndrome coronavirus 2 (SARS-CoV-2) was named "COVID-19" and characterized COVID-19 outbreak as a "pandemic" on March 11. As of June 8, 2020, nearly 7 million cases of COVID-19 have been reported globally, including more than four hundred thousand deaths[1].

The clinic presentation of COVID-19 varies remarkably, from asymptotic infection or mild illness to severe pneumonia with acute respiratory distress syndrome (ARDS) or death. Currently, there is still no Food and Drug Administration (FDA) approved drugs to specifically treat patients with COVID-19, although some drugs such as hydroxychloroquine approved for other indications, as well as multiple investigational agents, are being studied for the treatment of COVID-19 or be used for the hospitalized patients under an Emergency Use Authorization.

The natural history and prognosis of COVID-19 is not fully understood yet, however, there are increasing evidence indicating that lung fibrosis existed in the part of patients with COVID-19. In the patients with critical illness who receive lung transplantation or took autopsy after death, the obvious pulmonary fibrosis were observed in their lungs[2, 3]. After the nucleic acid (RNA) of SARS-Cov2 became negative or under detectable levels at 2 times of examination, these patients were considered as in "recovery phase". However, a lot of patients were not fully recovered actually. Almost $90 \%$ of cases in 90 Patients with COVID-19 Pneumonia had residual lesions by the computed tomography(CT) scan[4], and many patients had the short of breath since lung dysfunction in this recovery phase.SARS-CoV-2 has certain homology with SARS-COV[5], and the epidemiological characteristics of COVID-19 are also similar to SARS too. According to epidemic history of SARS, the survivors developed varying 
degrees of pulmonary fibrosis[6].The patients with COVID-19, in particular thoses with pneumonia may developed into fibrosis following pulmonary interstitial inflammation, which will impairs pulmonary function and quality of life[7, 8].

FuZheng HuaYu Tablet (FZHY), a patent herbal product for liver fibrosis, was approved by China National Medical Products Administration in 2002. FZHY consists of 6 herbs including Radix Salviae Miltiorrhizae, Cordyceps Mycelium Powder, Semen Persicae, Pollen Pini, Gynostemma Pentaphyllammak and Fructus Schisandrae Chinensis, and was manufactured with good quality of control in the dosage form of tablet and capsule. In our previous studies, FZHY was found to have a good effect on lung interstitial inflammation and fibrosis in the bleomycin induced rats[9-11]. Also a clinical observation showed that FZHY could obviously improve pulmonary function in the patients with chronic obstructive pulmonary disease (COPD)[12].

Given the patients with COVID-19 may have the lung fibrosis, the fibrosis in the different organs have similar or common pathological mechanisms, and FZHY shows an effect against lung fibrosis in animal and improve lung functions in patients with COPD, we design this multiple centers, randomized, placebo controlled clinic trail, where patients with pulmonary fibrosis and dysfunction due to COVID-19 in their recovery phase will receive FZHY or placebo. The main objective is to evaluate the efficacy and safety of FZHY on lung fibrosis and function in patients with COVID-19, which is expected to improve clinical prognosis.

\section{Methods/design}

This is a double-blind, parallel-group, placebo-controlled, 1 to 1 randomized, multicentric clinical superiority trial of orally administered FZHY versus placebo in patients with pulmonary fibrosis and respiratory impairment due to COVID-19 in their "recovery phase". Participants receive FZHY or placebo until they meet a primary endpoint event, the trial concludes, or they withdraw consent. Each patient will be observed for 24 weeks and followed up for 8 weeks. Follow-up of assessment measures will continue for the duration of the trial for those participants stopping trial medication due to adverse events or patient choice. The vital status and details of admission to hospital will be captured for all patients who have consented to participate in the trial regardless whether they have withdrawn from the intervention or trial assessments. 
Table 1

Time and Event Schedule

\begin{tabular}{|c|c|c|c|c|c|c|c|c|}
\hline \multirow[t]{2}{*}{ Item } & \multirow{2}{*}{ 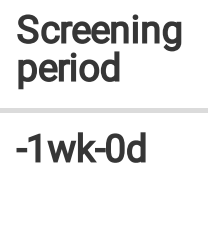 } & \multicolumn{6}{|c|}{ Treatment period } & \multirow{2}{*}{$\begin{array}{l}\text { Follow-up } \\
\text { period } \\
32 \mathrm{wk} \pm \\
7 \mathrm{~d}\end{array}$} \\
\hline & & $\begin{array}{l}4 w k \\
\pm 3 d\end{array}$ & $\begin{array}{l}8 w k \\
\pm 3 d\end{array}$ & $\begin{array}{l}12 w k \\
\pm 3 d\end{array}$ & $\begin{array}{l}16 w k \\
\pm 3 d\end{array}$ & $\begin{array}{l}20 w k \\
\pm 3 d\end{array}$ & $\begin{array}{l}24 \mathrm{wk} \\
\pm 3 d\end{array}$ & \\
\hline Eligibility Criteria & $\sqrt{ }$ & & & & & & & \\
\hline Sign informed consent & $\sqrt{ }$ & & & & & & & \\
\hline Demographic information & $\sqrt{ }$ & & & & & & & \\
\hline Past medical history & $\sqrt{ }$ & & & & & & & \\
\hline Symptoms & \multicolumn{8}{|c|}{ Recorded in APP everyday } \\
\hline Vital signs & $\sqrt{ }$ & $\sqrt{ }$ & $\sqrt{ }$ & $\sqrt{ }$ & $\sqrt{ }$ & $\sqrt{ }$ & $\sqrt{ }$ & $\sqrt{ }$ \\
\hline HRCT & $\sqrt{ }$ & $\sqrt{ }$ & $\sqrt{ }$ & $\sqrt{ }$ & & & $\sqrt{ }$ & $\sqrt{ }$ \\
\hline Lung function test & $\sqrt{ }$ & $\sqrt{ }$ & $\sqrt{ }$ & $\sqrt{ }$ & & & $\sqrt{ }$ & $\sqrt{ }$ \\
\hline 6-min walking test & $\sqrt{ }$ & $\sqrt{ }$ & $\sqrt{ }$ & $\sqrt{ }$ & $\sqrt{ }$ & $\sqrt{ }$ & $\sqrt{ }$ & $\sqrt{ }$ \\
\hline Dynamic oxygen saturat-ion & \multicolumn{8}{|c|}{ Wrist watch automatic recording and uploading daily } \\
\hline QOL-BREF & $\sqrt{ }$ & $\sqrt{ }$ & $\sqrt{ }$ & $\sqrt{ }$ & & & $\sqrt{ }$ & $\sqrt{ }$ \\
\hline PHQ-9 & $\sqrt{ }$ & $\sqrt{ }$ & $\sqrt{ }$ & $\sqrt{ }$ & & & $\sqrt{ }$ & $\sqrt{ }$ \\
\hline GAD-7 & $\sqrt{ }$ & $\sqrt{ }$ & $\sqrt{ }$ & $\sqrt{ }$ & & & $\sqrt{ }$ & $\sqrt{ }$ \\
\hline $\begin{array}{l}\text { SARS-CoV-2 specific IgG and } \\
\operatorname{lgM} \text { antibodies }\end{array}$ & $\sqrt{ }$ & & & $\sqrt{ }$ & & & $\sqrt{ }$ & $\sqrt{ }$ \\
\hline Routine blood test $\square$ CRP & $\sqrt{ }$ & & & $\sqrt{ }$ & & & $\sqrt{ }$ & $\sqrt{ }$ \\
\hline Routine urine test & $\sqrt{ }$ & & & $\sqrt{ }$ & & & $\sqrt{ }$ & $\sqrt{ }$ \\
\hline Stool-routine & $\sqrt{ }$ & & & $\sqrt{ }$ & & & $\sqrt{ }$ & $\sqrt{ }$ \\
\hline ECG & $\sqrt{ }$ & & & $\sqrt{ }$ & & & $\sqrt{ }$ & $\sqrt{ }$ \\
\hline Liver and kidney function & $\sqrt{ }$ & & & $\sqrt{ }$ & & & $\sqrt{ }$ & $\sqrt{ }$ \\
\hline Randomized grouping & $\sqrt{ }$ & & & & & & & \\
\hline $\begin{array}{l}\text { Dissemination of Drugs and } \\
\text { Participant Logs }\end{array}$ & $\sqrt{ }$ & $\sqrt{ }$ & $\sqrt{ }$ & $\sqrt{ }$ & $\sqrt{ }$ & $\sqrt{ }$ & $\sqrt{ }$ & \\
\hline $\begin{array}{l}\text { Recovery of residual drugs and } \\
\text { participant logs }\end{array}$ & & $\sqrt{ }$ & $\sqrt{ }$ & $\sqrt{ }$ & $\sqrt{ }$ & $\sqrt{ }$ & $\sqrt{ }$ & \\
\hline
\end{tabular}

The trial is set in 8 academic hospitals in three provinces or municipality in China. The first patient was randomized on April 14, 2020. In total, 160 patients will be recruited into the trial. This trial has ethical approval from IRB of Shuguang Hospital affiliated with Shanghai University of Traditional Chinese Medicine (Approval No. 2020-798-0501) and is registered onto Clinical Trials.gov(ID:NCT04279197). Ministry of science and technology of People's Republic of China is the trial sponsor. Professor Chenghai Liu is Principle Investigator (PI) for the clinic trial. A Trial 
Management Group (TMG) ,as shown in Table 2 , has been set up to assist with developing the design, coordination and strategic management of the trial. An independent Data Monitoring Committee (DMC) ,as shown in Table 2 ,has been established to provide oversight of safety and efficacy considerations, sample size estimation and study conduct. Following review, the DMC will recommend continuation, modification, or discontinuation of this study based on reported safety and efficacy data. Details of DMC responsibilities and procedures are specified in the DMC charter. Representatives of the sponsor will serve only as coordinators of the committee, without having full member responsibilities or privileges.

Table 2

Trial oversight committees

$\begin{array}{lll}\text { Name } & \text { Affiliation } & \text { Role and } \\ & \text { responsibilities }\end{array}$

\section{Trial Management Group}

\begin{tabular}{|c|c|c|}
\hline Professor Chenghai Liu & Shuguang Hospital Affiliated to Shanghai University of TCM & $\begin{array}{l}\text { Principal } \\
\text { investigator }\end{array}$ \\
\hline Dr.Feng Xing, Dr.Jing Lv & Shuguang Hospital Affiliated to Shanghai University of TCM & $\begin{array}{l}\text { Clinical trial } \\
\text { manager }\end{array}$ \\
\hline Yingchun $\mathrm{He}$ & Shanghai University of TCM & Statistician \\
\hline Dr.Zhimin Zhao & Shuguang Hospital Affiliated to Shanghai University of TCM & $\begin{array}{l}\text { Head of data } \\
\text { management }\end{array}$ \\
\hline Professor Wei Zhang & Shuguang Hospital Affiliated to Shanghai University of TCM & Co-investigator \\
\hline Professor Qi-wu Qiu & Jingmen No.1 People's Hospital & Co-investigator \\
\hline $\begin{array}{l}\text { Professor Xian-gao } \\
\text { Jiang }\end{array}$ & Wenzhou Central Hospital & Co-investigator \\
\hline Professor Jia Ke & Hubei Hospital of TCM & Co-investigator \\
\hline Professor Hui Wu & Wuhan N0.1 hospital & Co-investigator \\
\hline $\begin{array}{l}\text { Professor Peng-cheng } \\
\text { Luo }\end{array}$ & Wuhan N0.3 hospital & Co-investigator \\
\hline Professor Xin Zheng & $\begin{array}{l}\text { Union Hospital Affiliated to Tongji Medical College, Huazhong } \\
\text { University of Science and Technology }\end{array}$ & Co-investigator \\
\hline $\begin{array}{l}\text { Professor Jia-quan } \\
\text { Huang }\end{array}$ & $\begin{array}{l}\text { Tongji Hospital Affiliated to Tongji Medical College, Huazhong } \\
\text { University of Science and Technology }\end{array}$ & Co-investigator \\
\hline
\end{tabular}

Independent Data Monitoring Committee

\begin{tabular}{lll}
\hline Professor Ping Liu & Shanghai University of TCM & Chair \\
$\begin{array}{l}\text { Professor Qingshan } \\
\text { Zheng }\end{array}$ & Shanghai University of TCM & Statistician \\
\hline Dr.Yanyan Tao & Shuguang Hospital Affiliated to Shanghai University of TCM & Member \\
\hline
\end{tabular}

Neither the patients nor the researchers who participated in this trial are aware of the allocation. Drug on-site numbering is performed by statisticians who are unrelated to the trial. Drug packaging number, validation code and 
corresponding treatment group are generated by SAS software (version 9.4 or above).The drug packaging number and validation number are filled in (or pasted) on the label of the corresponding drug (test drug or control drug). The test drug is packed according to the visit period, and the drug for each visit period of the participants is packed as a package. Blinding record is saved during the process of compiling.

Researcher record data according to the content of case report form (CRF) and used the Electronic Clinical Data Management System (DAS for EDC V6.0) for electronic data management. The Data Management Plan (DMP), as a guiding document for data management, is written by the DMC and approved by the PI. The data management work will be carried out according to the time, content and method defined by the DMP. Data managers construct electronic case report forms (eCRFs) according to the design of the trial scheme and set up logical verification according to the data verification plan (DVP), which are released and used after passing the test and being approved by the PI. The eCRF data originated from the original records, and the data entry personnel filled in the instructions according to the eCRF, and timely entered the subject visit data into the Electronic Data Capture System (EDC). The Inspector checks the consistency of eCRF data and source data, and questions can be raised. After data entry is completed and Source Data Verification (SDV) is performed, the electronic signature verification is carried out by the researcher. If the data are revised after signature, it needs to be resigned. After the database lock record is signed jointly by the PI, sponsor, statistician and data manager, the data manager locks the database and submits the database to the statistician. Each participant's eCRF generates PDF electronic documents for preservation, and data managers write data management reports. After the statistical analysis was completed, the data manager closed the database.

Patient's information is recorded in the CRF. All the research results (including personal data, laboratory documents, etc.) appearing in the original medical records will be completely confidential within the limits permitted by law. The patient's name does not appear on the CRF, only the initials and assigned numbers appear. Only abbreviations and numbers of patients will appear in relevant research summaries, articles and public publications, if necessary. Drug supervision and administration departments, ethics committees or sponsor may consult the data of participating patients according to regulations. But without permission, they will not use the patient data for other purposes or leak it to other groups.

\section{Methods/design}

This is a double-blind, parallel-group, placebo-controlled, 1 to 1 randomized, multicentric clinical superiority trial of orally administered FZHY versus placebo in patients with pulmonary fibrosis and respiratory impairment due to COVID-19 in their "recovery phase". Participants receive FZHY or placebo until they meet a primary endpoint event, the trial concludes, or they withdraw consent. Each patient will be observed for 24 weeks and followed up for 8 weeks. Follow-up of assessment measures will continue for the duration of the trial for those participants stopping trial medication due to adverse events or patient choice. The vital status and details of admission to hospital will be captured for all patients who have consented to participate in the trial regardless whether they have withdrawn from the intervention or trial assessments.

Figure 1 provides a study flowchart of the trial design and Table 1 provides the time and event schedule. This trial protocol (v1.0, dated March 18, 2020) is based on the Standard Protocol Items: Recommendations for Interventional Trials (SPIRIT) 2013 Statement for protocols of clinical trials (see Additional file 3).

\section{Table 1 Time and Event Schedule}


Item

Screening Treatment period period

Follow-

Eligibility

Criteria

Sign informed $\sqrt{ }$

consent

Demographic $\sqrt{ }$

information

Past medical

history

\section{Symptoms Recorded in APP everyday}

$\begin{array}{lllllllll}\text { Vital signs } & \sqrt{ } & \sqrt{ } & \sqrt{ } & \sqrt{ } & \sqrt{ } & \sqrt{ } & \sqrt{ } & \sqrt{ } \\ \text { HRCT } & \sqrt{ } & \sqrt{ } & \sqrt{ } & \sqrt{ } & & & \sqrt{ } & \sqrt{ } \\ \begin{array}{l}\text { Lung function } \\ \text { test }\end{array} & \sqrt{ } & \sqrt{ } & \sqrt{ } & \sqrt{ } & & & \sqrt{ } & \sqrt{ } \\ \begin{array}{l}\text { 6-min walking } \\ \text { test }\end{array} & \sqrt{ } & \sqrt{ } & \sqrt{ } & \sqrt{ } & \sqrt{ } & \sqrt{ } & \sqrt{ } & \sqrt{ }\end{array}$

Dynamic Wrist watch automatic recording and uploading daily

oxygen

saturat-ion

QOL-BREF

PHQ-9

$\sqrt{ } \quad \sqrt{ }$

$\sqrt{ } \quad \sqrt{ }$

$\sqrt{ }$

$\begin{array}{ll}\sqrt{ } & \sqrt{ } \\ \sqrt{ } & \sqrt{ }\end{array}$

GAD-7

SARS-CoV-2

specific lgG

and $\lg \mathrm{M}$

antibodies

Routine blood $\quad \checkmark$

test $\ \mathrm{CRP}$

Routine urine $\quad \sqrt{ }$

test

Stool-routine

ECG

Liver and

kidney

function

Randomized

grouping

Dissemination $\sqrt{ }$

$\sqrt{ }$
$\sqrt{ }$
$\sqrt{ }$

$\sqrt{ }$

$\checkmark$

$\checkmark$

$\checkmark$

$\checkmark$

$\checkmark$

$\sqrt{ }$

$\checkmark$

$\checkmark$

(1)

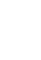


of Drugs and

Participant

Logs

Recovery of residual drugs

and

participant

logs

The trial is set in 8 academic hospitals in three provinces or municipality in China. The first patient was randomized on April 14, 2020. In total, 160 patients will be recruited into the trial. This trial has ethical approval from IRB of Shuguang Hospital affiliated with Shanghai University of Traditional Chinese Medicine (Approval No. 2020-798-0501) and is registered onto Clinical Trials.gov(ID:NCT04279197). Ministry of science and technology of People's Republic of China is the trial sponsor. Professor Chenghai Liu is Principle Investigator (PI) for the clinic trial. A Trial Management Group (TMG) ,as shown in Table 2 , has been set up to assist with developing the design, coordination and strategic management of the trial. An independent Data Monitoring Committee (DMC) ,as shown in Table 2 ,has been established to provide oversight of safety and efficacy considerations, sample size estimation and study conduct. Following review, the DMC will recommend continuation, modification, or discontinuation of this study based on reported safety and efficacy data. Details of DMC responsibilities and procedures are specified in the DMC charter. Representatives of the sponsor will serve only as coordinators of the committee, without having full member responsibilities or privileges.

\section{Table 2 Trial oversight committees}


Name

Affiliation

Role and

responsibilities

\section{Trial Management Group}

Professor Chenghai Liu

Shuguang Hospital Affiliated to Shanghai University of TCM

Principal

investigator

Dr.Feng Xing, Dr.Jing Lv Shuguang Hospital Affiliated to Shanghai University of TCM $\begin{aligned} & \text { Clinical trial } \\ & \text { manager }\end{aligned}$

$\begin{array}{lll}\text { Yingchun He } & \text { Shanghai University of TCM } & \text { Statistician } \\ \text { Dr.Zhimin Zhao } & \text { Shuguang Hospital Affiliated to Shanghai University of TCM } & \begin{array}{r}\text { Head of data } \\ \text { management }\end{array}\end{array}$

\begin{tabular}{|lll}
\hline Professor Wei Zhang & Shuguang Hospital Affiliated to Shanghai University of TCM & Co-investigator \\
\hline Professor Qi-wu Qiu & Jingmen No.1 People's Hospital & Co-investigator \\
\hline $\begin{array}{l}\text { Professor Xian-gao } \\
\text { Jiang }\end{array}$ & Wenzhou Central Hospital & Co-investigator \\
\hline Professor Jia Ke & Hubei Hospital of TCM & Co-investigator \\
\hline Professor Hui Wu & Wuhan N0.1 hospital & Co-investigator \\
\hline $\begin{array}{l}\text { Professor Peng-cheng } \\
\text { Luo }\end{array}$ & Wuhan N0.3 hospital & Co-investigator \\
\hline
\end{tabular}
Professor Xin Zheng Union Hospital Affiliated to Tongji Medical College, Huazhong Co-investigator University of Science and Technology
Professor Jia-quan Tongji Hospital Affiliated to Tongji Medical College, Huazhong Co-investigator Huang University of Science and Technology

\section{Independent Data Monitoring Committee}

\begin{tabular}{lll} 
Professor Ping Liu & Shanghai University of TCM & Chair \\
\hline $\begin{array}{l}\text { Professor Qingshan } \\
\text { Zheng }\end{array}$ & Shanghai University of TCM & Statistician \\
\hline Dr.Yanyan Tao & Shuguang Hospital Affiliated to Shanghai University of TCM & Member
\end{tabular}

Neither the patients nor the researchers who participated in this trial are aware of the allocation. Drug on-site numbering is performed by statisticians who are unrelated to the trial. Drug packaging number, validation code and corresponding treatment group are generated by SAS software (version 9.4 or above).The drug packaging number and validation number are filled in (or pasted) on the label of the corresponding drug (test drug or control drug). The test drug is packed according to the visit period, and the drug for each visit period of the participants is packed as a package. Blinding record is saved during the process of compiling.

Researcher record data according to the content of case report form (CRF) and used the Electronic Clinical Data Management System (DAS for EDC V6.0) for electronic data management. The Data Management Plan (DMP), as a guiding document for data management, is written by the DMC and approved by the PI. The data management work will be carried out according to the time, content and method defined by the DMP. Data managers construct electronic case report forms (eCRFs) according to the design of the trial scheme and set up logical verification 
according to the data verification plan (DVP), which are released and used after passing the test and being approved by the PI. The eCRF data originated from the original records, and the data entry personnel filled in the instructions according to the eCRF, and timely entered the subject visit data into the Electronic Data Capture System (EDC). The Inspector checks the consistency of eCRF data and source data, and questions can be raised. After data entry is completed and Source Data Verification (SDV) is performed, the electronic signature verification is carried out by the researcher. If the data are revised after signature, it needs to be resigned. After the database lock record is signed jointly by the PI, sponsor, statistician and data manager, the data manager locks the database and submits the database to the statistician. Each participant's eCRF generates PDF electronic documents for preservation, and data managers write data management reports. After the statistical analysis was completed, the data manager closed the database.

Patient's information is recorded in the CRF. All the research results (including personal data, laboratory documents, etc.) appearing in the original medical records will be completely confidential within the limits permitted by law. The patient's name does not appear on the CRF, only the initials and assigned numbers appear. Only abbreviations and numbers of patients will appear in relevant research summaries, articles and public publications, if necessary. Drug supervision and administration departments, ethics committees or sponsor may consult the data of participating patients according to regulations. But without permission, they will not use the patient data for other purposes or leak it to other groups.

\section{Patient characteristics}

Patients need to meet the following inclusion and exclusion criteria:

$\underline{\text { Inclusion criteria }}$

- Compliance with the diagnostic criteria for COVID-19 (China Diagnosis and Treatment Protocol for COVID19(Trial Version 7);

- Real-time fluorescent polymerase chain reaction test to detect of SARS-CoV-2 nucleic acid in respiratory specimens or blood specimens of patients with negative results ( $>2$ times);

- Pulmonary CT scans within 7 days showed that there were still unabsorbed inflammation or pulmonary fibrosis in the lungs;

- Age 18-70;

- Six-minute walk distance less than 350 meters;

- Participate in clinical research voluntarily and sign informed consent.

\section{Exclusion criteria}

- Patients who have undergone lung surgery that affects pulmonary function, such as pulmonary transplantation, pulmonary resection, pulmonary volume reduction, etc;

- Relying on mechanical ventilation to maintain pulmonary function, such as ventilators;

- Combined with chronic pulmonary diseases affecting pulmonary function, such as chronic obstructive pulmonary disease, other known causes of interstitial pulmonary disease;

- Patients with diseases affecting cardiac function, such as pulmonary circulation hypertension, heart failure, peripheral vascular disease, fibromyalgia, and pacemaker installation; 
- Patients with severe underlying diseases affecting survival, including uncontroled cardiac, renal, digestive, hematological, neuropsychiatric, immune, metabolic diseases, malignant diseases and severe malnutrition;

- Resting heart rate $>120$ times/min;

- Systolic blood pressure > $180 \mathrm{mmHg}$, diastolic blood pressure > $100 \mathrm{mmHg}$;

- Unstable angina pectoris or myocardial infarction occurring within the last month;

- Severe obesity $\left(\mathrm{BMl}>30 \mathrm{~kg} / \mathrm{m}^{2}\right)$;

- Allergic constitution, allergic to the drug components involved in the treatment program;

- Pregnant or breastfeeding women;

- Patients with disabilities who are unable to complete the efficacy evaluation questionnaires;

- Difficult collaborators with poor mental health status, suffering from mental illness, patients without selfcontrol, unable to express clearly;

- Those who are participating in other clinical trials;

- According to the investigator's judgment, patients whose enrollment complications or poor compliance will affect the efficacy and safety evaluation.

\section{Identification, Recruitment}

Patients will be mainly recruited from 8 hospitals, as shown in Table 3 . These hospitals are all comprehensive hospitals and can carry out diagnostic and therapeutic activities for convalescent patients with COVID-19. Potential recruits are being approached by local clinic teams and provided with Informed consent and given at least 24 hours to read this prior to consent. Consent is taken by appropriately-trained clinicians or delegated members of staff.

\section{Table 3 Participating sites}

\begin{tabular}{|ll|}
\hline Trust name & Principal investigator \\
\hline Shuguang Hospital Affiliated to Shanghai University of TCM & Professor Wei Zhang \\
\hline Jingmen No.1 People's Hospital & Professor Qi-wu Qiu \\
\hline Wenzhou Central Hospital & $\begin{array}{l}\text { Professor Xian-gao } \\
\text { Jiang }\end{array}$ \\
\hline Hubei Hospital of TCM & Professor Jia Ke \\
\hline Wuhan NO.1 hospital & Professor Hui Wu \\
\hline $\begin{array}{l}\text { Wuhan NO.3 hospital } \\
\begin{array}{l}\text { Union Hospital Affiliated to Tongji Medical College, Huazhong University of Science } \\
\text { and Technology }\end{array}\end{array}$ & $\begin{array}{l}\text { Professor Peng-cheng } \\
\text { Luo }\end{array}$ \\
\hline $\begin{array}{l}\text { Tongji Hospital Affiliated to Tongji Medical College, Huazhong University of Science Xin Zheng } \\
\text { and Technology }\end{array}$ & $\begin{array}{l}\text { Professor Jia-quan } \\
\text { Huang }\end{array}$ \\
\hline
\end{tabular}

\section{Randomization}

A double-blind study design is chosen to preserve study integrity and minimize bias in the assessment of all study endpoints. After patients have provided their written informed consent, completed all Screening assessments and 
received confirmation of eligibility, they will be 1:1 randomization into the study using an Interactive Web Randomization System (IWRS) and stratification based on:

- Age: $\geq 50$ years vs. $<50$ years

- Pulmonary inflammation: Chest CT shows that there is still acute exudative disease in the lung, but it had been significantly improved vs no acute exudative disease in the lung

- 6-minute walk test: $\geq 250 \mathrm{~m}$ vs. $<250 \mathrm{~m}$

These stratification factors are chosen on the basis that they may be sufficiently prognostic such that an imbalance may bias the results. Participants, caregivers, and the study team assessing the outcomes are blinded to group assignment.

\section{Interventions}

The two groups will be given the same basic treatment, but all of them are restricted to anti-fibrosis herbs or medications. The experimental group is given FZHY, and the control group is given placebo of FZHY, which appearance, taste, quality, taking times and methods are the same as those of FZHY. Basic treatment includes respiratory function rehabilitation training and Vitamin $\mathrm{C}$ tablets. Detailed interventions are shown in Table 3.

\section{Table4 Grouping and specific interventions}

\begin{tabular}{|lll|}
\hline Groups & Interventions & Remarks \\
\hline$\underline{\text { Control }}$ & $\begin{array}{l}\text { basic treatment (respiratory function } \\
\text { rehabilitation training + Vitamin C } \\
\text { tablets) }\end{array}$ & $\begin{array}{l}\text { Vitamin C tablets, administration: } 0.2 \mathrm{~g} / \text { time, } 3 \text { times/ } \\
\text { day, oral }\end{array}$ \\
& + placebo of FZHY & $\begin{array}{l}\text { Placebo of FZHY or FZHY, administration: } 0.4 \mathrm{~g} / \text { tablet, } \\
1.6 \mathrm{~g} / \text { time, } 3 \text { times/day, oral; medicine to be taken after } \\
\text { meals. }\end{array}$ \\
$\begin{array}{ll}\text { Experimental } \\
\text { group }\end{array}$ & $\begin{array}{l}\text { basic treatment (same as control } \\
\text { group) + FZHY }\end{array}$ & \\
\hline
\end{tabular}

\section{Primary outcomes}

The primary outcome for the trial is a composite endpoint consisting of lung function (pulmonary function test, 6minute walk test) and high resolution computed tomography (HRCT).

- Pulmonary function test

Pulmonary function test is a commonly used method for evaluating pulmonary function in clinical practice. We need to detect forced vital capacity (FVC), percentage of FVC to the predicted value, forced expiratory volume in 1 second (FEV1), carbon monoxide diffusion (DLCO) in this trial. Spirometry[13] and DLCO[14] are being measured according to current American Thoracic Society/European Respiratory Society guidelines. FVC and DLCO are both components of prognostic modelling algorithms[15], are frequently utilised in clinical trials[16].

- The 6-minute walk test

The 6-minute walk test is a common method to objectively evaluate the functional exercise capacity of patients with moderate to severe pulmonary disease[17]. In this study, the longest 6-minute walking distance of patients is measured, and the lowest oxygen saturation of the six-minute walking test is collected before the study. patients are 
required to wear wearable equipment, and are monitored remotely every day. When pulmonary function returned to normal, monitoring is stopped. The lowest oxygen saturation is recorded after each six-minute walking test is completed.

- HRCT

HRCT is a commonly used method to identify pulmonary fibrosis in clinic, research proves that HRCT may be useful for assessing the risk of acute exacerbation of idiopathic pulmonary fibrosis (AE-IPF) in patients with IPF, independent of ethnicity[18], HRCT pulmonary fibrosis area and pneumonia severity index (PSI) are observed.

\section{Secondary outcomes}

Secondary efficacy criteria includes dynamic oxygen saturation, discomfort symptoms, quality of life assessment, and SARS-CoV-2-specific IgG and IgM antibodies.

- Blood oxygen saturation

Blood oxygen saturation is an important physiological parameter in the process of respiratory circulation, and the change of blood oxygen saturation can reflect the state of lung function. In this study, the lowest oxygen saturation of the six-minute walk test is collected before the test. Wearable equipment is worn during the test. The monitoring is stopped when the pulmonary function returned to normal. The lowest oxygen saturation is recorded when the sixminute walk test is completed.

- Discomfort symptoms

Discomfort symptoms include dyspnea, cough, exhausted, fatigue, insomnia, sweating, poor appetite, diarrhea, etc., which are common manifestations of patients with COVID-19[19,20]. And, they are also a common clinical manifestation of respiratory diseases. In this study, the 0-10 visual analogue score method is used to record the most severe symptom score and the average score within 24 hours; before the study and during the study, the patients' APP is used to record the symptoms daily.

- Quality of life assessment

Quality of Life-BREF (QOL-BREF)[21], Patient Health Questionnaire-9(PHQ-9)[22] and Generalized anxiety disorder7(GAD-7)[23] can all reflect the quality of life of patients to some extent.

- SARS-CoV-2-specific IgG and IgM antibody detection

Virus-specific antibody detection is one of the important means for laboratory diagnosis of viral diseases. Antibody detection is of great significance for virus diagnosis and time of infection, evaluation of the infection stage of the virus and prediction of disease outcome.

\section{End Points}

The primary efficacy end points are pulmonary function recovery and fibrosis reverse rates assessed on the 4, 8,12 , and 24-week period.

In this trial, definition of pulmonary function recovery is: pulmonary function test normalized(FVC $\mathrm{FEV} 1 \square$

DLCO $\geq 80 \%$ predictive value, FEV1/FVC $\geq 92 \%$ predictive value ,or meet the following three requirements at the same 
time:(1) Lowest Blood oxygen saturation $\geq 95 \%$ on two consecutive days, (2) 6MWD>500m, (3) pneumonia absorbed totally in HRCT.

Pulmonary fibrosis reverse means that HRCT reveal fibrosis vanished in lungs.

The main secondary end points are the absolute change from baseline in the 6MWD at week 24 and time to pulmonary function recovery.

\section{Safety outcomes}

Blood routine, urine routine, fecal routine, electrocardiogram and liver囚kidney function assessment is being taken at baseline, 12,24 weeks and Week 8 follow-up.

\section{Adverse events}

Researchers will assess the risk of patients throughout the study. If serious adverse events occur, they should report to the responsible unit of the project in time and take corresponding measures to remedy them. If a change in drug dosage is required due to moderate or serious adverse events (clinical and/or laboratory tests), the researcher should make a request, and the drug dose can only be changed if interrupted or adjusted according to the comprehensive judgement of the expert committee. When subsequent adverse events improve, a further dose increase may be considered. These changes must be documented in the CRF. In addition, patients may be allowed to withdraw for any reason, but all data collection required by the protocol at the time of withdrawal must be completed as far as possible.

\section{Sample size}

Because there is no reference to calculate the sample size, this trial presupposes a sample size of 160 cases (Including 20\% shedding rate) according to the research experience, 80 cases in each group. During the trial, the sample size may be increased appropriately or the course of trial may be adjusted after evaluation and discussion by the DMC.

\section{Data management}

Clinical data management will be performed by the sponsor designee according to procedures described in a comprehensive data management plan. The data management plan will include procedures for processing the data from this study, and will describe the responsibilities of the sponsor and designee when clinical data management is provided by an external vendor. In particular, the data management plan will include a list of the standard operating procedures that apply to this study.

\section{Statistical analysis}

The modified intent-to-treat (mITT) analysis set will be considered as the full analysis set. This will include all evaluable patients, ie, all randomized patients who receive at least one dose of Fuzheng Huayu or placebo and who have lung function test at randomization and at least one post randomization lung function test.

In general, all demographics, baseline characteristics, efficacy and safety variables will be summarized using descriptive statistics and graphs as appropriate. Continuous variables will be summarized by descriptive statistics 
(sample size (n), mean, standard deviation (SD), minimum, median and maximum). Categorical variables will be summarized in frequency tables (frequencies and percentages).

The primary efficacy endpoint of the study is the lung function recovery rate. We will summarize recovery rate using a binomial response rate and corresponding two-sided 95\% exact confidence interval (Cl) as per the ClopperPearson method. At each visit, odds ratio (OR) (FZHY vs. Placebo) with 95\% Cl will be reported, logistic regression will utilized adjusted for potential confounders, age, baseline 6-minute walk test, Pulmonary inflammation and site.

The change in 6-minute walking distance from baseline to week 24 is secondary efficacy parameter. The analysis will be conducted using a mixed-effects model for repeated-measures (MMRM) approach with treatment group, study site, visit and treatment group $\mathrm{x}$ visit interaction as fixed effects and the baseline distance and baseline $\mathrm{x}$ visit interaction as the covariates.

Safety analyses will be performed in all treated subjects. Descriptive statistics of safety will be presented using National Cancer Institute (NCI) Common Terminology Criteria for Adverse Events (CTCAE) version 5.0. All TEAEs, Grade 3-4 AEs, treatment-related AEs, Grade 3-4 treatment-related AEs, SAEs, treatment-related SAEs, and AEs leading to discontinuation will be tabulated using worst grade per NCI CTCAE v 5.0 criteria by system organ class and preferred term.

All statistical analyses will be performed using SAS version 9.4. Tests of statistical significance for all efficacy endpoint analyses will use the 2-sided significance level of 0.05 .

\section{Adaptive designs}

An interim analysis is planed when 60 subjects completed the 4-week study by IDMC. If 4 weeks lung function recovery rate is more than $60 \%$, then compare the lung function recovery rate of the two groups at 4 weeks. Sample size estimation will be based on interim results.

The adaptive design could be based on a 4-week measurement of patient lung function state when the primary endpoint is the assessment of the same outcome at 24 weeks.

The flexibilities offered by adaptive designs may be particularly useful in this exploratory period of development by allowing initial evaluation of a broad range of choices. Using adaptive designs in this trial to learn about patient's early stage response offers sponsors opportunities that can improve the designs and shorter expected calendar time.

There is no requirement to adjust for multiplicity due to interim analyses, since there are no planned interim analyses with the opportunity to make an early claim of efficacy.

\section{Dissemination}

The results of this study regardless of the direction of effect, will form research reports, academic papers and dissertations, which will be submitted to government departments and peer review. In addition, results will be published in relevant journals or international conferences.

\section{Discussion}


The pandemic COVID-19 is new health crisis threatening the world, although its natural history is not fully understood yet, with a lower mortality rate than SARS, the type and severity of complications in the severe cases are similar to SARS, and the pneumonia and pulmonary fibrosis could be occurred in some patients[24-26].

The pulmonary fibrosis, caused by kinds of etiologies, could impair respiratory function. There is still short of effective agents against pulmonary fibrosis, although it was reported that Pirfenidone and Nintedanib could be effective for IPF[27-30], which have disadvantages such as expensive price and severe side effects. Therefore, it is very important to develop the agent to inhibit or regress the pulmonary fibrosis, especially for one duo to COVID-19.

FZHY is a botanic product composed of 6 herbs, with an indication for liver fibrosis approved by China FDA in 2002. Our clinic study approved that FZHY could regress liver fibrosis due to hepatitis B virus[31], and the clinic trial in US indicated that FZHY could stabilize and regress the liver fibrosis due to hepatitis $\mathrm{C}$ virus[32]. Also the action mechanism of FZHY against liver fibrosis were investigated, which were associated with protection of hepatocyte inflammation, inhibition of stellate cell activation, and regulation of hepatic matrix metabolism[33].

The fibrosis in different organs had common features[34], such as activation of fibroblasts and deposition of extracellular matrix. The evidence showed that the subepithelial basement membrane often destroyed, which play a pivot role in the pathological development of pulmonary interstitial fibrosis, while matrix metalloproteinase (MMP) regulate the degradation and remodeling of the extracellular matrix of the basement membrane[35]. In our animal experiments, it was found that FZHY could decreased active MMP-2 activity in the fibrotic lungs induced by BLM, and indicating that FZHY could improve the lung inflammation and fibrosis through protection of subepithelial basement membrane. Therefore, it is rationale for us to apply FZHY for pulmonary fibrosis due to COVID -19.

Hereto, we focus on the COVID-19 patients with pulmonary fibrosis in their recovery phase, which means the patients become negative SASR-2 RNA, but still suffer from pulmonary fibrosis and/or dysfunctions after quarantine and treatments. And these patients will receive FZHY or placebo, in order to evaluate the effectiveness of FZHY on the pulmonary fibrosis and dysfunction due to SRSA-2.

The primary endpoint is lung fibrosis, the golden diagnostic method is liver function measured by meter, the specific indicators include FVC, FEV1\%, DLCO, etc. however, many hospitals ban this machine use due to the possible contamination and virus spread. HRCT is another option to detect the pulmonary fibrosis and pneumonia, which can find small, local lesions and ensures accurate analysis of lesion characteristics and distribution based on cross-sectional images and multiplanar reconstruction[36], and semi-quantization also could obtained with image analysis and algorithm. Meantime, we will monitor the oxygen saturation with wearable devices and observe the health-related quality of life as the secondary endpoint, the latter is easy to be accomplished by the participants unlike the St. George's Respiratory Questionnaire[37], which would need at least 10 minutes and not easy to complete without help.

Sample size and duration are very important aspects for clinic trial, however there is no prior experience and references given COVID-19 is a novel disease. Therefore, we adopt the adaptive design for the trial. Although we preset the sample size of 80 each group, considering general population of 60 patients each arm for moderated size and $20 \%$ of withdrawal rate, the sample could adjust (increase or stop enrollment) when the efficacy is evaluated at the middle course of the trial (12 week) DMC. And the individual duration for observation also is flexible, and the patient could terminate the trial in advance if he or she satisfy the normalized criteria including negative HRCT, Six-minute walk distance $>500 \mathrm{~m}$.

Page 16/21 
Although FZHY is much safe for patients with chronic liver diseases, the safety assessment will be fully observed. All participants will receive the compensatory payment for their transformation and nutrition in addition to free examinations and treatments.

In short, in view of the characteristics of pulmonary interstitial inflammation and fibrosis in patients with COVID-19 during recovery period, this study plans to establish an effective treatment program for pulmonary fibrosis/dysfunction in COVID-19 to promote the rehabilitation of patients. The study results would not only provide new methods for the treatment of pulmonary fibrosis due to COVID-19 and information for further understanding the natural history of COVID-19, but provide a potential strategy for treating pulmonary fibrosis caused by other etiologies.

\section{Abbreviations}

acute exacerbation of idiopathic pulmonary fibrosis: AE-IPF; acute respiratory distress syndrome: ARDS; Case report Form: CRF; Carbon monoxide diffusion: DLCO; Chronic obstructive pulmonary disease: COPD; Computed tomography: CT; Common Terminology Criteria for Adverse Events: CTCAE; confidence interval: Cl; Data management plan: DMP; Data manager: DM; Data monitoring committee: DMC; Data verification plan: DVP; Drug Analysis System for Interactive Web Response System: DAS for IWRS; electronic case report forms: eCRF; Electronic Clinical Data Management System : DAS for EDC; Electronic Data Capture System: EDC; Food and Drug Administration: FDA; Forced expiration volume in 1 second: FEV1; Forced vital capacity: FVC; Generalized anxiety disorder: GAD; High resolution computed tomography: HRCT; Matrix metalloproteinase: MMP; mixed-effects model for repeated-measures: MMRM; modified intent-to-treat: mITT; National Cancer Institute: NCl; odds ratio: OR; Patient health questionnaire: PHQ; Pneumonia severity index: PSI; Principle Investigator: PI; Quality of Life-BREF: QOLBREEF; Severe acute respiratory syndrome: SARS; Source data verification: SDV; Trial management group: TMG; World Health Organization: WHO.

\section{Declarations}

\section{Trial status:}

Protocol version: 1.0, dated March 18, 2020. Recruitment is ongoing, started April 14, 2020 and anticipated end date is December 31, 2021.

\section{Acknowledgements}

The authors are thankful for the ongoing support from all our principal investigators, site staff and trial participants in undertaking various aspects of the trial. We would also like to express our gratitude to those who reviewed the protocol including: Trial Management Group and Data Monitoring Committee.

\section{Funding}

This trial is supported by the Ministry of science and Technology (CN) Plan "Major special projects of the Ministry of science and Technology (CN)" (No.2020yfc084500). The funder did not participate in the design of the study and collection, analysis, and interpretation of data and in writing the manuscript.

\section{Availability of data}

Page 17/21 
Requests for access to trial data will be considered, and approved in writing where appropriate, after formal application to the TMG.

\section{Authors' contributions}

F J drafted this paper. All authors contributed to revisions of the manuscript, read and approved the final manuscript.

\section{Ethics approval and consent to participate}

IRB of Shuguang Hospital affiliated with Shanghai University of TCM (Approval No. 2020-798-05-01) has approved this trial. Participant consent is obtained prior to any trial-related procedure. During the consent process it is made clear that the participant can decline to participate in all or any aspect of the trial, at any time and for any reason, without affecting their future care or treatment. Patients unable to provide written informed consent are deemed ineligible for the trial.

\section{Consent for publication}

Not applicable.

\section{Competing interests}

The authors of this paper have no financial or other competing interests that impact on their responsibilities towards the scientific value or potential publishing activities associated with the trial.

\section{References}

1. World Health Organization. https://covid19.who.int/. Accessed 8 June 2020.

2. Luo W, Yu H, Gou J, Li X, Sun Y, Li J, Liu L. Clinical Pathology of Critical Patient with Novel Coronavirus Pneumonia (COVID-19). Preprints 2020, 2020020407.

3. Tian S, et al. Pulmonary Pathology of Early-Phase 2019 Novel Coronavirus (COVID-19) Pneumonia in Two Patients With Lung Cancer. J Thorac Oncol. 2020;15(5):700-4.

4. Wang Y, et al. Temporal Changes of CT Findings in 90 Patients with COVID-19 Pneumonia: A Longitudinal Study. Radiology. 2020:200843.

5. Roujian, Lu, et al. Genomic characterisation and epidemiology of 2019 novel coronavirus: implications for virus origins and receptor binding. The Lancet. 2020;395(10224):565-74.

6. Venkataraman T, Coleman CM, Frieman MB. Overactive Epidermal Growth Factor Receptor Signaling Leads to Increased Fibrosis after Severe Acute Respiratory Syndrome Coronavirus Infection. J Virol. 2017;91(12):00182-17.

7. Zumla A, Hui DS, Azhar El, Memish ZA, Maeurer M. Reducing mortality from 2019-nCoV: host-directed therapies should be an option. Lancet. 2020;395(10224):e35-e6.

8. Liu J, et al. Overlapping and discrete aspects of the pathology and pathogenesis of the emerging human pathogenic coronaviruses SARS-CoV, MERS-CoV, and 2019-nCoV. J Med Virol. 2020;92(5):491-4.

9. Weicheng Y, Xiong Lu, Ping L, et al. Therapeutic Effect of Fuzheng Huayu Recipe on Bleomycin-induced Pulmonary Interstitial Fibrosis in Rats. JTCM. 2006;47(7):538-40. 
10. Tan SZ, et al. Effect of Fuzheng Huayu Recipe on MMP-2 activity and type IV collagen expression in fibrotic lung tissue of rats. Chinese Journal of Materia Medica. 2007;32(0):835-8.

11. Yang K, et al. Herbal Compatibility of Fuzheng Huayu Recipe against Pulmonary Fibrosis. JTCM. 2009;50(8):732-6.

12. Zhang W, et al. Research on Fuzheng Huayu Capsule treating chronic obstruct pulmonary in clinic. Liaoning Journal of TCM. 2008;36(8):1174-5.

13. Miller MR, et al. Standardisation of spirometry. Eur Respir J. 2005;26(2):319-38.

14. Zavorsky GS, et al. Standardisation and application of the single-breath determination of nitric oxide uptake in the lung. Eur Respir J. 2017;49(2).

15. du Bois RM, et al. Ascertainment of individual risk of mortality for patients with idiopathic pulmonary fibrosis. Am J Respir Crit Care Med. 2011;184(4):459-66.

16. King TE, et al. A phase 3 trial of pirfenidone in patients with idiopathic pulmonary fibrosis. $\mathrm{N}$ Engl $\mathrm{J}$ Med. 2014;370(22):2083-92.

17. Agarwala P, Salzman SH. Six-Minute Walk Test: Clinical Role, Technique, Coding, and Reimbursement. Chest. 2020;157(3):603-11.

18. Hirano C, et al. Baseline High-Resolution CT Findings Predict Acute Exacerbation of Idiopathic Pulmonary Fibrosis: German and Japanese Cohort Study. J Clin Med. 2019;8(12).

19. Hui DSC, Zumla A. Severe Acute Respiratory Syndrome: Historical, Epidemiologic, and Clinical Features. Infect Dis Clin North Am. 2019;33(4):869-89.

20. Huang C, et al. Clinical features of patients infected with 2019 novel coronavirus in Wuhan, China. Lancet. 2020;395(10223):497-506.

21. WHO Quality of Life-BREF (WHOQOL-BREF). http://www.who.int/substance_abuse/research_tools/whoqolbref/en/. Accessed 8 June 2020.

22. Kroenke K, Spitzer RL, Williams JB. The PHQ-9: validity of a brief depression severity measure. J Gen Intern Med. 2001;16(9):606-13.

23. Spitzer RL, Kroenke K, Williams JB, Löwe B. A brief measure for assessing generalized anxiety disorder: the GAD-7. Arch Intern Med. 2006;166(10):1092-7.

24. Ngai JC, et al. The long-term impact of severe acute respiratory syndrome on pulmonary function, exercise capacity and health status. Respirology. 2010;15(3):543-50.

25. Hui DS, et al. The 1-Year Impact of Severe Acute Respiratory Syndrome on Pulmonary Function, Exercise Capacity, and Quality of Life in a Cohort of Survivors. Chest. 2005;128(4):2247-61.

26. Marchioni A, et al. Acute exacerbation of idiopathic pulmonary fibrosis: lessons learned from acute respiratory distress syndrome? Crit Care. 2018;22(1):80.

27. Cottin V, Maher T. Long-term clinical and real-world experience with pirfenidone in the treatment of idiopathic pulmonary fibrosis. Eur Respir Rev. 2015;24(135):58-64.

28. Hanta I, Cilli A, Sevinc C. The Effectiveness, Safety, and Tolerability of Pirfenidone in Idiopathic Pulmonary Fibrosis: A Retrospective Study. Adv Ther. 2019;36(5):1126-31.

29. Tepede A, Yogaratnam D. Nintedanib for Idiopathic Pulmonary Fibrosis. J Pharm Pract. 2019;32(2):199-206.

30. Crestani B, et al. Long-term safety and tolerability of nintedanib in patients with idiopathic pulmonary fibrosis: results from the open-label extension study, INPULSIS-ON. Lancet Respir Med. 2019;7(1):60-8. 
31. Wang T, et al. Fuzheng Huayu capsule as an adjuvant treatment for HBV-related cirrhosis: A systematic review and meta-analysis. Phytother Res. 2018;32(5):757-68.

32. Tarek H, et al. Sa1319 A Phase II, Randomized, Placebo-Controlled, Double-Blind, Multi-Center Study to Assess the Antifibrotic Activity of Fuzheng Huayu in Chronic Hepatitis C Patients With Hepatic Fibrosis (S-USIIT-01). Gastroenterology. 2014;146(5).

33. Liu C, Hu Y, Xu L, Liu C, Liu P. Effect of Fuzheng Huayu formula and its actions against liver fibrosis. Chin Med. 2009;4:12.

34. Majo J, Klinkhammer BM, Boor P, Tiniakos D. Pathology and natural history of organ fibrosis. Curr Opin Pharmacol. 2019;49:82-9.

35. Gueders MM, Foidart JM, Noel A, Cataldo DD. Matrix metalloproteinases (MMPs) and tissue inhibitors of MMPs in the respiratory tract: potential implications in asthma and other lung diseases. Eur J Pharmacol. 2006;533:133-44.

36. Raghu G, et al. Diagnosis of Idiopathic Pulmonary Fibrosis. An Official ATS/ERS/JRS/ALAT Clinical Practice Guideline. Am J Respir Crit Care Med. 2018;198(5):e44-68.

37. Furukawa T, et al. The St. George's Respiratory Questionnaire as a prognostic factor in IPF. Respir Res. 2017;18(1):18.

\section{Figures}

\section{Convalescent patients with COVID-19( $n=160)$}

Viral nucleic acid negative $\geq 2$ times、Pulmonary fibrosis and/or pulmonary dysfunction

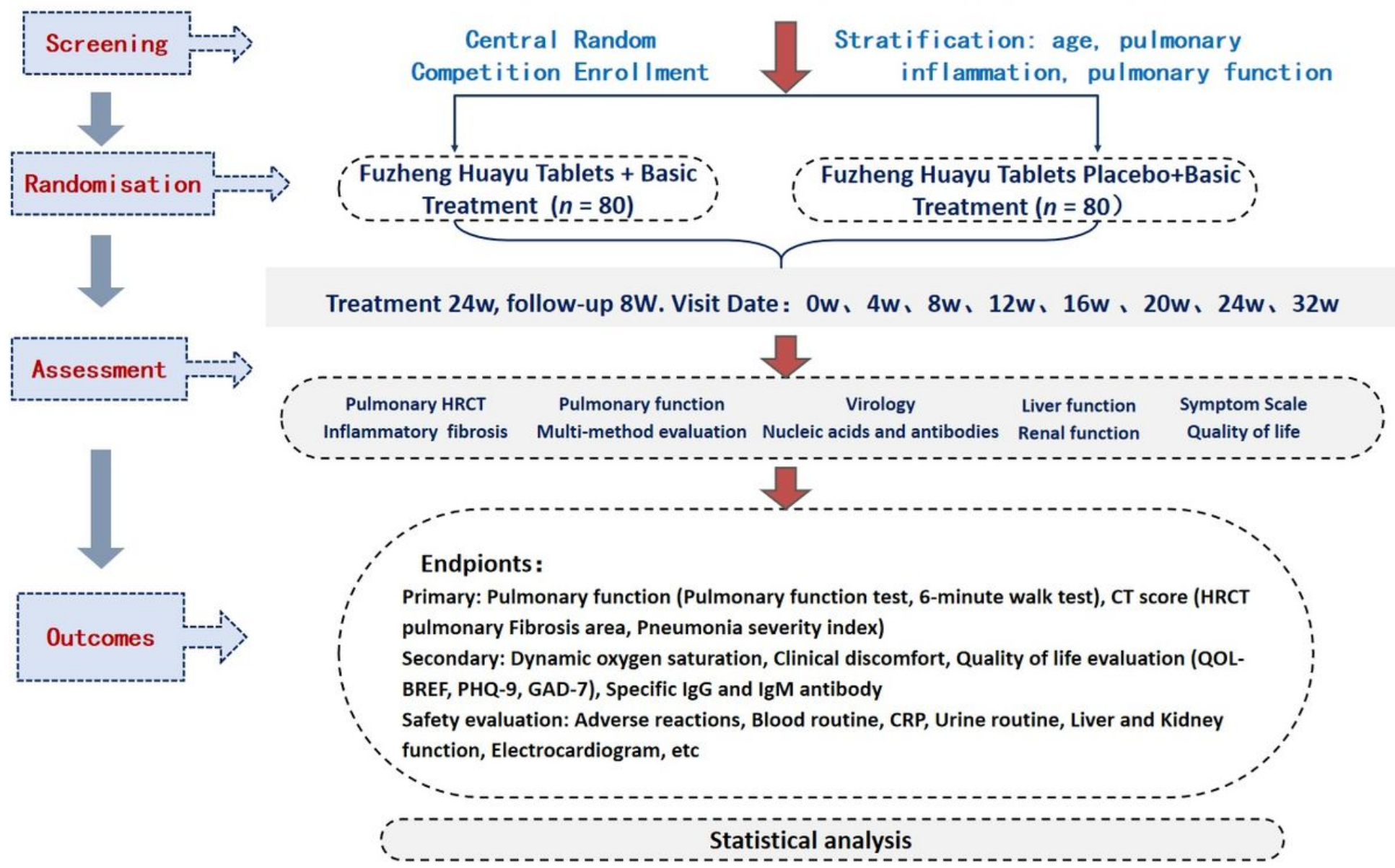


Figure 1

Study Flowchart

\section{Supplementary Files}

This is a list of supplementary files associated with this preprint. Click to download.

- Funding.pdf

- Ethicsapproval.pdf

- SPIRIT2013Checklist0620.doc 